RECORDS OF PHARMACEUTICAL
AND BIOMEDICAL SCIENCES

\title{
Cytotoxic and Antileishmanial Activities of the Red Sea Soft Coral Sarcophyton glaucum Extract and Some of Its Isolates
}

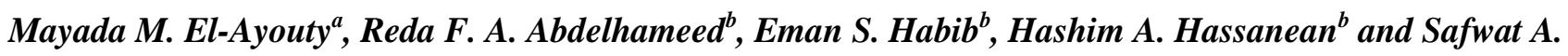 \\ Ahmed $^{b *}$. \\ ${ }^{a}$ Department of Pharmacognosy, Faculty of Pharmacy, Sinai University, El-Arish 45511, Egypt; ${ }^{b}$ Department \\ of Pharmacognosy, Faculty of Pharmacy, Suez Canal University, Ismailia 41522, Egypt.
}

\section{Received on: 2-8-2021}

Revised on: 29-8-2021

Accepted on: 3-9-2021

Correspondence Author:

Tel: +20-010-92638387

E-mail address:

safwat_aa@yahoo.com

\begin{abstract}
Truthfully, natural products have been the principal productive source of advancing new drugs. The broad aim of this work was to conduct a phytochemical and biological study of the Red Sea soft coral Sarcophyton glaucum, Family: Alcyoniidae collected from Red Sea at the Egyptian coasts. The phytochemical investigation from the soft coral S. glaucum led to the isolation of ten compounds including: palmitic acid (1), stearic acid (2), (24S)-24-methyl cholesterol (3), batyl alcohol (4), heptadecanoic acid pentadecyl ester (5), sarcophine (6), (+)-7 $\alpha, \quad 8 \beta$ dihydroxydeepoxysarcophine (7), uracil (8), thymine (9) and a ceramide (10). As cancer is one of the most hazardous factors threatening the human life, in this study the potential in-vitro cytotoxicity of the soft coral $S$. glaucum extract and three marine isolates were measured against HepG2 and MCF7 using Sulphorhodamine-B (SRB) assay and all of the tested samples showed a good cytotoxic activities against both hepatic and breast cancer. The extract of $S$. glaucum was tested also against the protozoan parasite Leishmania donovani, using pentamidine and amphotericin B as controls and showed antileishmanial activity.
\end{abstract}

Keywords: Sarcophyton glaucum; cytotoxic activity; antileishmanial activity.

\section{Introduction:}

Marine life forms, comprising more than half of the entire worldwide organisms, offer an important source of possibly novel active compounds. Soft corals comprising an important group of marine organisms. There are about 35 species belonging to the soft coral genus Sarcophyton, and they are hard to be distinguished (Verseveldt, 1982). The soft coral Sarcophyton is a good source of different structures of diterpenes of cembrane type, sesquiterpenes, steroids, fatty acids and amino acids (Jia et al., 2006, Lan et al., 2007). Soft corals synthesize terpenoids as compounds used for chemical defense to avoid predatory fishes which attack their soft bodies (Iwagawa et al., 1999). So, many secondary metabolites isolated from soft corals have been proved to possess many different biological activities such as anti-microbial, anti-fungal, anti-tumor, anti-viral and antiinflammatory (Wei et al., 2013).

In our study we are trying to discover biologically active compounds from Egyptian natural products, our group in focused on the isolation of the active compounds from the soft coral S. glaucum, and identification of the isolated compounds using different spectroscopic techniques. The potential in- 
vitro cytotoxicity of the soft coral S. glaucum extract and three of the isolated compounds were measured against HepG2 and MCF7 and the extract was tested also against the protozoan parasite Leishmania donovani, using pentamidine and amphotericin $\mathrm{B}$ as controls.

\section{Materials and Methods}

\subsection{Animal material:}

About $5 \mathrm{Kg}$ of $S$. glaucum were collected by hand using SCUBA from Safaga at the Egyptian Red Sea. It was immediately frozen and kept at $\left(-24^{\circ} \mathrm{C}\right)$. A voucher specimen was deposited in the Egyptian Red Sea invertebrates collection at the Department of Pharmacognosy, Suez Canal University under registration number (SAA-54). The identification and description of the soft coral was provided by Dr. Tarek A. Temraz, Department of Marine Science, Faculty of Science, Suez Canal University, Ismailia, Egypt.

\subsection{Extraction and fractionation of the soft coral and isolation of pure compounds:}

The soft coral $(5 \mathrm{~kg})$ of the frozen chopped small pieces were extracted with $\mathrm{MeOH}-\mathrm{CH}_{2} \mathrm{Cl}_{2}$ (1:1) (5L $\times 4$ ) at room temperature to yield $200 \mathrm{~g}$ of dry extract. Fractionation using vacuum liquid chromatography (VLC) with gradient elution led to separation of different fractions. Using $n$-hexane, EtOAc, and $\mathrm{MeOH}$ gradient elution, F2 fraction (20 g) $(25 \%$ EtOAc in hexane) was subjected to column chromatography of silica gel type, two fractions were obtained and subjected to different chromatographic separation techniques, including silica gel leading to isolation of $\mathbf{1}(20 \mathrm{mg}), \mathbf{2}(15 \mathrm{mg})$ and $\mathbf{3}(8 \mathrm{mg})$. Fractions $\mathrm{F} 3$ and $\mathrm{F} 4$ of 50\%+75\% EtOAc in hexane, $38 \mathrm{~g}$ was purified using silica gel, three subfractions were obtained and subjected to different chromatographic separation techniques, including sephadex LH-20 leading to isolation of $\mathbf{4}(30 \mathrm{mg}), \mathbf{5}$ $(14 \mathrm{mg}) 6(50 \mathrm{mg})$ and $7(15 \mathrm{mg})$. F5 Fraction of $100 \%$ EtOAc, $21 \mathrm{~g}$ was purified using silica gel column chromatography and sephadex LH-20 to yield $8(20 \mathrm{mg})$ and $\mathbf{9}(15 \mathrm{mg}) .27 \mathrm{~g}$ of fraction F6 (5\% $\mathrm{MeOH}$ in EtOAc) was purified using silica gel column chromatography and sephadex LH-20 to yield $10(25 \mathrm{mg})$.

2.3. In-vitro cytotoxicity of the soft coral $S$. glaucum extract and some of its isolates:
The potential in-vitro cytotoxicity of the soft coral $S$. glaucum extract and the isolated compounds 6,7 and 10 was measured against HepG2 and MCF7 using Sulphorhodamine-B (SRB) assay following the method reported by Vichai and Kirtikara (Vichai and Kirtikara, 2006).

\subsection{In-vitro antileishmanial assay of the soft} coral $S$. glaucum extract:

The extract was tested against the protozoan parasite Leishmania donovani, using pentamidine and amphotericin B as controls using the method of (Bharate et al., 2008; Jain et al., 2005).

\section{Results and discussion}

\subsection{Identification and characterization of the isolated compounds:}

The structure elucidation of the isolated compounds was deduced on the basis of spectroscopic methods, (El-MS, 1D and 2D NMR), physicochemical properties in addition to comparison with literature data and/or authentic samples. The isolated compounds were identified as:

Compound 1 was identified as palmitic acid (Figure 1) (Di Pietro et al., 2020), as molecular formula was determined to be $\mathrm{C}_{16} \mathrm{H}_{32} \mathrm{O}_{2}$ by El-mass spectrum as the molecular ion peak appeared at $\mathrm{m} / \mathrm{z} 256.08[\mathrm{M}]^{+}$. ${ }^{1} \mathrm{H}-\mathrm{NMR}\left(\mathrm{CD}_{3} \mathrm{OD}-\mathrm{CDCl}_{3}\right): \delta 2.32(2 \mathrm{H}, \mathrm{m}, \mathrm{H}-2)$, $1.63(2 \mathrm{H}, m, \mathrm{H}-3), 1.27$ (24H, s, H-4: H-15), 0.84 $(3 \mathrm{H}, t, J=8.0, \mathrm{H}-16) .{ }^{13} \mathrm{C}-\mathrm{NMR}\left(\mathrm{CD}_{3} \mathrm{OD}-\mathrm{CDCl}_{3}\right): \delta$ 173.8 (C-1), 34.3 (C-2), 31.8 (C-3), 22.6-29.6 (C-4: C-15), 14.0 (C-16).

Compound 2 was identified as stearic acid (Error! Reference source not found.) (Di Pietro et al., 2020). molecular formula was determined to be $\mathrm{C}_{18} \mathrm{H}_{36} \mathrm{O}_{2}$ by El-mass spectrum as the molecular ion peak appeared at $\mathrm{m} / \mathrm{z} \quad 284.21 \quad[\mathrm{M}]^{+} .{ }^{1} \mathrm{H}-\mathrm{NMR}$ $\left(\mathrm{CD}_{3} \mathrm{OD}-\mathrm{CDCl}_{3}\right): \delta 2.35(2 \mathrm{H}, m, \mathrm{H}-2), 1.64(2 \mathrm{H}, m$, $\mathrm{H}-3), 1.27$ (28H, $s, \mathrm{H}-4: \mathrm{H}-17), 0.89$ (3H, $t, J=8.0$, $\mathrm{H}-18) .{ }^{13} \mathrm{C}-\mathrm{NMR}\left(\mathrm{CD}_{3} \mathrm{OD}-\mathrm{CDCl}_{3}\right): \delta 179.9(\mathrm{C}-1)$, 33.9 (C-2), 31.8 (C-3), 22.6-29.6 (C-4: C-17), 14.0 (C-18).

Compound 3 was identified as (24S)-24-methyl cholesterol (Figure 1) (Rahelivao et al., 2017), molecular formula was determined to be $\mathrm{C}_{28} \mathrm{H}_{48} \mathrm{O}$ by El-MS spectrum $\left(\mathrm{m} / \mathrm{z} \quad 400.26 \quad\left[\mathrm{M}^{+}\right]\right) .{ }^{1} \mathrm{H}-\mathrm{NMR}$ $\left(\mathrm{CD}_{3} \mathrm{OD}-\mathrm{CDCl}_{3}\right): \delta 0.93(1 \mathrm{H}, m, \mathrm{H}-1 \mathrm{a}), 1.86(1 \mathrm{H}, m$, $\mathrm{H}-1 \mathrm{~b}), 1.93(1 \mathrm{H}, m, \mathrm{H}-2 \mathrm{a}), 1.54(1 \mathrm{H}, m, \mathrm{H}-2 \mathrm{~b}), 3.50$ $(1 \mathrm{H}, m, \mathrm{H}-3), 2.23(2 \mathrm{H}, m, \mathrm{H}-4), 5.35(1 \mathrm{H}, m, \mathrm{H}-6)$, $2.01(1 \mathrm{H}, m, \mathrm{H}-7 \mathrm{a}), 1.13(1 \mathrm{H}, m, \mathrm{H}-7 \mathrm{~b}), 0.87(1 \mathrm{H}$, $m, \mathrm{H}-8), 1.07(1 \mathrm{H}, m, \mathrm{H}-9), 0.93$ (2H, $m, \mathrm{H}-11), 1.20$ 


\section{El-Ayouty et al.}

$1.33(2 \mathrm{H}, m, \mathrm{H}-12), 1.20(1 \mathrm{H}, m, \mathrm{H}-14), 0.93(2 \mathrm{H}$, $m, \mathrm{H}-15), 1.38$ (1H, $m, \mathrm{H}-17), 0.67$ (3H, $s, \mathrm{H}-18)$, $1.01(3 \mathrm{H}, s, \mathrm{H}-19), 1.82(1 \mathrm{H}, m, \mathrm{H}-20 \mathrm{a}), 1.86(1 \mathrm{H}$, $m, \mathrm{H}-20 \mathrm{~b}), 0.91(3 \mathrm{H}, m, \mathrm{H}-21), 1.26(1 \mathrm{H}, m, \mathrm{H}-22 \mathrm{a})$, $1.82(1 \mathrm{H}, m, \mathrm{H}-22 \mathrm{~b}), 1.19(1 \mathrm{H}, m, \mathrm{H}-23 \mathrm{a}), 1.56(1 \mathrm{H}$, $m, \mathrm{H}-23 \mathrm{~b}), 1.58$ (1H, $m, \mathrm{H}-24), 1.50(1 \mathrm{H}, m, \mathrm{H}-25)$, $0.77(3 \mathrm{H}, m, \mathrm{H}-26), 0.85$ (3H, $m, \mathrm{H}-27), 0.68(3 \mathrm{H}, m$, $\mathrm{H}-28) .{ }^{13} \mathrm{C}-\mathrm{NMR}\left(\mathrm{CD}_{3} \mathrm{OD}-\mathrm{CDCl}_{3}\right): \delta 37.3(\mathrm{C}-1)$, 31.6 (C-2), 71.7 (C-3), 42.3(C-4), 140.8 (C-5), 121.6 (C-6), 31.9 (C-7), 31.9 (C-8), 50.1 (C-9), 36.5 (C10), 21.1 (C-11), 39.8 (C-12), 42.3 (C-13), 56.6 (C14), 24.3 (C-15), 28.2 (C-16), 56.0 (C-17), 11.8 (C18), 19.4 (C-19), 36.2 (C-20), 18.9 (C-21), 33.7 (C22), 30.6 (C-23), 39.1 (C-24), 31.5 (C-25), 17.6 (C26), 20.5 (C-27), 15.4 (C-28).

Compound 4 was identified as batyl alcohol (Figure 1) (Sun et al., 2015), molecular formula was determined to be $\mathrm{C}_{21} \mathrm{H}_{44} \mathrm{O}_{3}$ by El-MS spectrum as $\mathrm{m} / \mathrm{z}$ $345.21[\mathrm{M}+\mathrm{H}]^{+} .{ }^{1} \mathrm{H}-\mathrm{NMR}\left(\mathrm{CD}_{3} \mathrm{OD}-\mathrm{CDCl}_{3}\right): \delta 3.67$ $(1 \mathrm{H}, d d, J=3.6,11.4 \mathrm{~Hz}, \mathrm{H}-1 \mathrm{a}), 3.58(1 \mathrm{H}, d d, J=5.4$, $11.4 \mathrm{~Hz}, \mathrm{H}-1 \mathrm{~b}), 3.84(1 \mathrm{H}, m, \mathrm{H}-2), 3.43(2 \mathrm{H}, m, \mathrm{H}-$ $3), \quad 3.43\left(2 \mathrm{H}, m, \mathrm{H}-1^{\prime}\right), 1.55\left(2 \mathrm{H}, m, \mathrm{H}-2^{\prime}\right), 1.25$ $\left(30 \mathrm{H}\right.$, brs, H-3 : $\left.\mathrm{H}-17^{`}\right), 0.85(3 \mathrm{H}, t, J=6.6 \mathrm{~Hz}, \mathrm{H}-$ 18). ${ }^{13} \mathrm{C}-\mathrm{NMR}\left(\mathrm{CD}_{3} \mathrm{OD}-\mathrm{CDCl}_{3}\right): \delta 71.8(\mathrm{C}-1)$, 72.3(C-2), 64.2 (C-3), 70.6 (C-1`), 31.9 (C-2`), 22.6:29.7 (C-3`:C17`), 14.0 (C-18`).

Compound 5 was identified as heptadecanoic acid pentadecyl ester (Figure 1) (Pedpradab et al., 2012), molecular formula was determined to be $\mathrm{C}_{32} \mathrm{H}_{64} \mathrm{O}_{2}$ by El-MS spectrum as $m / z 480.55[\mathrm{M}+\mathrm{H}]^{+}$. ${ }^{1} \mathrm{H}-\mathrm{NMR}\left(\mathrm{CD}_{3} \mathrm{OD}-\mathrm{CDCl}_{3}\right): \delta 4.04(2 \mathrm{H}, t, J=6.8 \mathrm{~Hz}$, H-1 $), 2.28(2 \mathrm{H}, t, J=6.4 \mathrm{~Hz}, \mathrm{H}-2), 1.60(4 \mathrm{H}, m, \mathrm{H}-3$, H-2`), 1.26 (50H, $\left.s, \mathrm{H}-4: \mathrm{H}-16, \mathrm{H}-3{ }^{`}: \mathrm{H}-14{ }^{`}\right), 0.84$ $\left.(6 \mathrm{H}, t, J=6.8, \mathrm{H}-17, \mathrm{H}-15)^{\prime}\right){ }^{13} \mathrm{C}-\mathrm{NMR}\left(\mathrm{CD}_{3} \mathrm{OD}-\right.$ $\left.\mathrm{CDCl}_{3}\right): \delta 174.1(\mathrm{C}-1), 64.5\left(\mathrm{C}-1^{`}\right), 34.5(\mathrm{C}-2), 32.0$ (C-3, C-2`), 22.8-29.8 (C-4:C-16, C-3`:C-14`), 14.2 (C-17, C-15).

Compound $\mathbf{6}$ was identified as sarcophine (Figure 1) (Abdel-Wahhab et al., 2012), molecular formula was determined to be $\mathrm{C}_{20} \mathrm{H}_{28} \mathrm{O}_{3}$ by HRESIMS as $\mathrm{m} / z$ $317.2142[\mathrm{M}+\mathrm{H}]^{+} .{ }^{1} \mathrm{H}-\mathrm{NMR}\left(\mathrm{CD}_{3} \mathrm{OD}-\mathrm{CDCl}_{3}\right): \delta$ $5.51(1 \mathrm{H}, d d, J=10.0,1.5 \mathrm{~Hz}, \mathrm{H}-2), 4.97(1 \mathrm{H}, d d$, $J=10.0,1.0 \mathrm{~Hz}, \mathrm{H}-3), 2.30$ (2H, $m, \mathrm{H}-5), 1.63(2 \mathrm{H}$, $m, \mathrm{H}-6), 2.61(1 \mathrm{H}, m, \mathrm{H}-7), 1.03,1.86(2 \mathrm{H}, m, \mathrm{H}-9)$, $2.14(2 \mathrm{H}, m, \mathrm{H}-10), 5.07(1 \mathrm{H}, b r t, J=5.1 \mathrm{~Hz}, \mathrm{H}-11)$, $1.98(2 \mathrm{H}, m, \mathrm{H}-13), 2.69$ (2H, $m, \mathrm{H}-14), 1.78(3 \mathrm{H}, s$, $\mathrm{H}-17), 1.83$ (3H, $s, \mathrm{H}-18), 1.21$ (3H, $s, \mathrm{H}-19), 1.55$ $(3 \mathrm{H}, s, \mathrm{H}-20) .{ }^{13} \mathrm{C}-\mathrm{NMR}\left(\mathrm{CD}_{3} \mathrm{OD}-\mathrm{CDCl}_{3}\right): \delta 162.3$ (C-1), 78.6 (C-2), 120.4 (C-3), 143.8 (C-4), 37.2 (C5), 25.1 (C-6), 61.2 (C-7), 59.8 (C-8), 38.8 (C-9),

\section{Rec. Pharm. Biomed. Sci. 5 (2), 115-120, 2021}

23.1 (C-10), 124.7 (C-11), 135.4 (C-12), 36.2 (C13), 27.4 (C-14), 122.7 (C-15), $174.6(\mathrm{C}-16), 8.8$ (C17), 17.0 (C-18), 16.0 (C-19), 15.3(C-20).

Compound 7 was identified as (+)-7 $\alpha, 8 \beta$ dihydroxydeepoxysarcophine (Figure 1), (Wei Bie et al., 2008), molecular formula was determined to be $\mathrm{C}_{20} \mathrm{H}_{30} \mathrm{O}_{4}$ by HRESIMS as $m / z 335.2180[\mathrm{M}+\mathrm{H}]^{+}$. ${ }^{1} \mathrm{H}-\mathrm{NMR}\left(\mathrm{CD}_{3} \mathrm{OD}-\mathrm{CDCl}_{3}\right): \delta 5.79(1 \mathrm{H}, d, J=10.2$ $\mathrm{Hz}, \mathrm{H}-2), 4.85(1 \mathrm{H}, d, J=10.2 \mathrm{~Hz}, \mathrm{H}-3), 2.30(1 \mathrm{H}$, $m, \mathrm{H}-5 \mathrm{a}), 2.02(1 \mathrm{H}, m, \mathrm{H}-5 \mathrm{~b}), 1.84(1 \mathrm{H}, m, \mathrm{H}-6 \mathrm{a})$, 1.29 (1H, $m, \mathrm{H}-6 \mathrm{~b}), 3.29(1 \mathrm{H}, d, J=10.5 \mathrm{~Hz}, \mathrm{H}-7)$, 1.73 (1H. $m, \mathrm{H}-9 \mathrm{a}), 1.46(1 \mathrm{H} . d t, J=9.9,9.5 \mathrm{~Hz}, \mathrm{H}-$ 9b), 2.16(1H, $m, \mathrm{H}-10 \mathrm{a}), 1.97(1 \mathrm{H}, m, \mathrm{H}-10 \mathrm{~b}) 5.10$ $(1 \mathrm{H}, d d, J=6.5,6.7 \mathrm{~Hz}, \mathrm{H}-11), 2.16(1 \mathrm{H}, m, \mathrm{H}-13 \mathrm{a})$, 2.04(1H, $m, \mathrm{H}-13 \mathrm{~b}), 2.65(1 \mathrm{H}, m, \mathrm{H}-14 \mathrm{a}), 2.01(1 \mathrm{H}$, $m, \mathrm{H}-14 \mathrm{~b}), 1.76$ (3H, $s, \mathrm{H}-17), 1.81$ (3H, $s, \mathrm{H}-18)$, $0.99(3 \mathrm{H}, s, \mathrm{H}-19), 1.59(3 \mathrm{H}, s, \mathrm{H}-20) .{ }^{13} \mathrm{C}-$ $\mathrm{NMR}\left(\mathrm{CD}_{3} \mathrm{OD}-\mathrm{CDCl}_{3}\right): \delta 163.2(\mathrm{C}-1), 79.3(\mathrm{C}-2)$, 120.7 (C-3), 144.2 (C-4), 35.4 (C-5), 26.9 (C-6), 72.7 (C-7), 75.6 (C-8), 36.9 (C-9), 23.6 (C-10), 125.3 (C-11), 134.7 (C-12), 36.5 (C-13), 26.8 (C14), 122.6 (C-15), 175.2 (C-16), 8.9 (C-17), 16.5 (C18), 24.2 (C-19), 15.3 (C-20).

Compound 8 was identified as uracil (Figure 1) (Sun et al., 2015). ${ }^{1} \mathrm{H}-\mathrm{NMR}\left(\mathrm{CD}_{3} \mathrm{OD}-\mathrm{CDCl}_{3}\right): \delta$ 11.00(1H, brs, NH-1), 10.80 (1H, brs, NH-3), 5.41 $(1 \mathrm{H}, d, J=7.6 \mathrm{~Hz}, \mathrm{H}-5), 7.35(1 \mathrm{H}, d, J=7.6 \mathrm{~Hz}, \mathrm{H}-$ 6). ${ }^{13} \mathrm{C}-\mathrm{NMR}\left(\mathrm{CD}_{3} \mathrm{OD}-\mathrm{CDCl}_{3}\right): \delta 152.1(\mathrm{C}-2), 164.9$ (C-4), 100.8 (C-5), 142.8 (C-6).

Compound 9 was identified as thymine (Figure 1) (Sun et al., 2015). ${ }^{1} \mathrm{H}-\mathrm{NMR}\left(\mathrm{CD}_{3} \mathrm{OD}-\mathrm{CDCl}_{3}\right): \delta$ $10.54(1 \mathrm{H}, b r s, \mathrm{NH}-1), 10.95(1 \mathrm{H}$, brs, $\mathrm{NH}-3), 7.23$ $(1 \mathrm{H}, s, \mathrm{H}-6), 1.72(3 \mathrm{H}, s, \mathrm{H}-7) .{ }^{13} \mathrm{C}-\mathrm{NMR}\left(\mathrm{CD}_{3} \mathrm{OD}-\right.$ $\left.\mathrm{CDCl}_{3}\right): \delta 151.5(\mathrm{C}-2), 164.9$ (C-4), 107.7 (C-5), 137.7 (C-6), 11.8 (C-7).

Compound 10 was identified as a ceramide (Figure 1) (Abdelhameed et al., 2018). The mass spectrum showed a molecular ion peak at $\mathrm{m} / \mathrm{z}\left(706[\mathrm{M}+\mathrm{Na}]^{+}\right.$, $\left.684[\mathrm{M}+\mathrm{H}]^{+}\right)$, according to the results of $\mathbf{1 0}$ methanolysis with methanolic hydrochloric acid followed by EI-MS analysis of 10 FAME, afforded a molecular ion peak at $\mathrm{m} / \mathrm{z} 398[\mathrm{M}]^{+}$, indicating $\mathrm{C} 23$ fatty acid methyl esters. ${ }^{1} \mathrm{H}-\mathrm{NMR}\left(\mathrm{C}_{5} \mathrm{D}_{5} \mathrm{~N}\right): \delta 8.58$ $(1 \mathrm{H}, d, J=8.9 \mathrm{~Hz}, \mathrm{NH}), 4.43(1 \mathrm{H}, d d, J=10.8,5.2 \mathrm{~Hz}$, $\mathrm{H}-1 \mathrm{a}), 4.49(1 \mathrm{H}, d d, J=10.8,4.6 \mathrm{~Hz}, \mathrm{H}-1 \mathrm{~b}), 5.12$ $(1 \mathrm{H}, m, \mathrm{H}-2), 4.35(1 \mathrm{H}, m, \mathrm{H}-3), 4.27(1 \mathrm{H}, m, \mathrm{H}-4)$, $4.61\left(1 \mathrm{H}, m, \mathrm{H}-2^{\prime}\right), 0.86\left(6 \mathrm{H}, m,-\mathrm{CH}_{3}\right)$,

$1.27\left(s,\left(-\mathrm{CH}_{2}\right)_{\mathrm{n}}\right) .{ }^{13} \mathrm{C}-\mathrm{NMR}\left(\mathrm{C}_{5} \mathrm{D}_{5} \mathrm{~N}\right): \delta 62.0(\mathrm{C}-1)$, 52.9 (C-2), 76.7 (C-3), 73.0 (C-4), 175.2 (C-1`) 72.4 $\left(\mathrm{C}-2{ }^{\prime}\right), 14.2\left(\mathrm{CH}_{3}\right), 22.4\left(\mathrm{CH}_{2}\right)$.

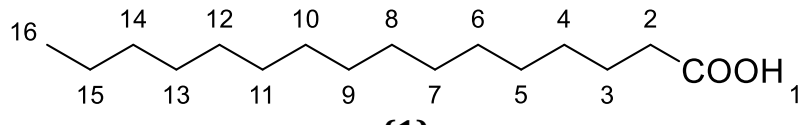

(1) 


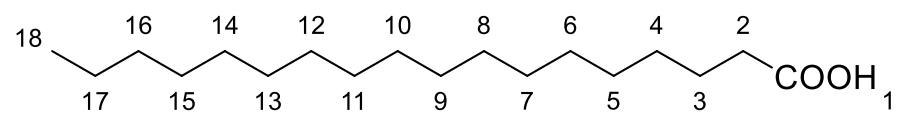

(2)

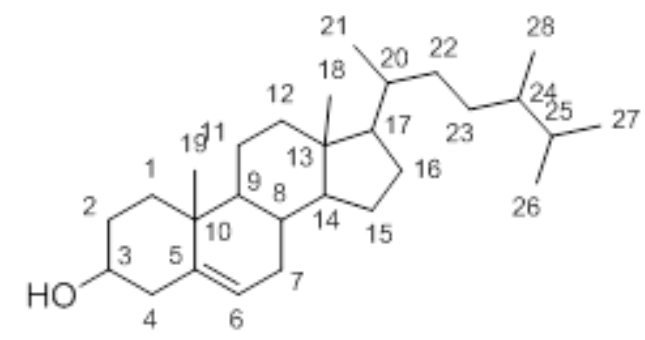

(3)

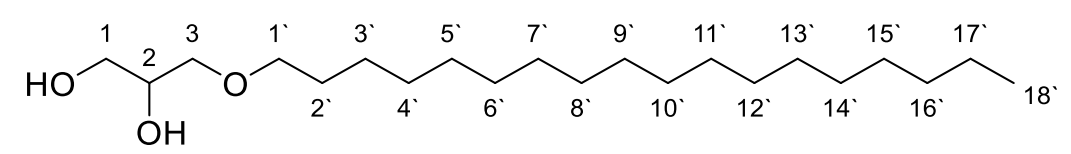

(4)

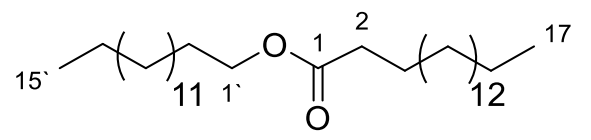

(5)

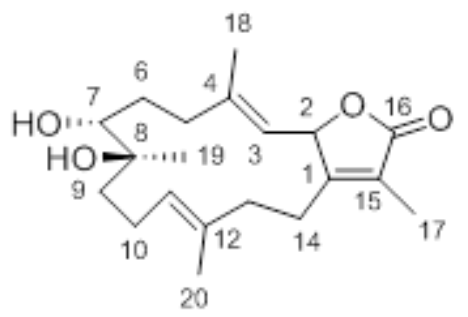

(7)

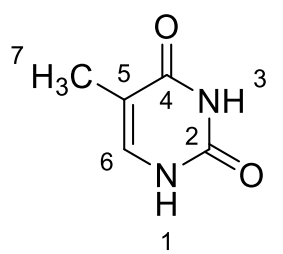

(9)

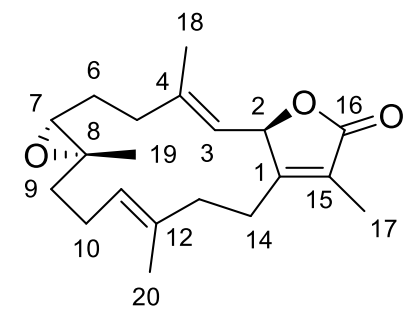

(6)

(8)

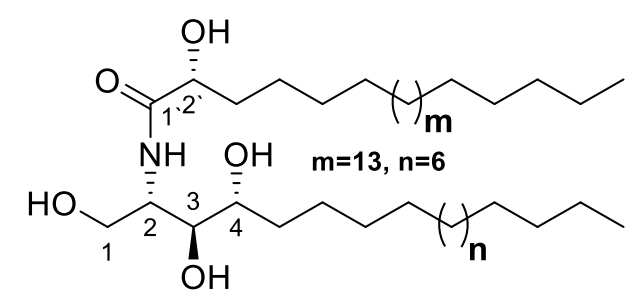

(10)

Figure (1): Isolated compounds from S. glaucum extract.

Table 1: In-vitro cytotoxic effects $\left(\mathrm{IC}_{50}, \mu \mathrm{g} / \mathrm{mL}\right)$ of $S$. glaucum extract and some of isolated compounds on HepG2 and MCF7 cell lines. 


\begin{tabular}{|c|c|c|}
\hline Sample & HepG2 & MCF7 \\
\hline Extract & 19.3 & 18.7 \\
\hline 6 & 10.4 & 8.33 \\
\hline 7 & 4.88 & 3.98 \\
\hline 10 & 10.5 & 11 \\
\hline Doxo & 4.2 & 3.83 \\
\hline
\end{tabular}

Table 2: Antileishmanial screening assays of the soft coral $S$. glaucum extract.

\begin{tabular}{|l|l|lc|}
\hline Sample & \multicolumn{1}{|c|}{ IC $_{50} \boldsymbol{\mu g} / \mathbf{m L}$} & IC90 $\boldsymbol{\mu g} / \mathbf{m L}$ \\
\hline Extract & 100 & $>100$ & \\
\hline
\end{tabular}

$\mathrm{IC}_{50}$ : concentration causing $50 \%$ growth inhibition.

$\mathrm{IC}_{90}$ : concentration causing $90 \%$ growth inhibition.

\subsection{In-vitro cytotoxicity of the soft coral $S$. glaucum extract and some of its isolates:}

Screening of the total extract of $S$. glaucum and isolated compounds 6,7 and 10 resulted in promising cytotoxic activities against MCF-7 and HepG2 cell lines when compared to Doxorubicin as illustrated in (Table 1).

\subsection{In-vitro antileishmanial assay of the soft coral $S$. glaucum extract:}

The extract was tested against the protozoan parasite Leishmania donovani, using pentamidine and amphotericin B as controls (Table 2) .This test indicated that this marine has antileishmanial activity.

\section{Conclusion}

The phytochemical examination of the soft coral $S$. glaucum including isolation and identification of: palmitic acid (1), stearic acid (2), (24S)-24-methyl cholesterol (3), batyl alcohol (4), heptadecanoic acid pentadecyl ester (5), sarcophine (6) (+)-7 $\alpha, 8 \beta$ dihydroxydeepoxysarcophine (7), uracil (8), thymine (9) and ceramide (10). the soft coral $S$. glaucum extract and compounds 6, 7 and 10 showed good anticancer activities against both hepatic and breast cancer. The extract of $S$. glaucum has antileishmanial activity.

\section{Conflict of interest}

The authors report no declaration of conflict of interest.

Jain, M., Khan, S.I., Tekwani, B.L., Jacob, M.R., Singh, S., Singh, P.P., Jain, R. 2005. Synthesis, antimalarial, antileishmanial, and antimicrobial activities of some 8-quinolinamine analogues.

\section{Funding}

This work was funded by the authors.

\section{References}

Abdelhameed, R., Ibrahim, A., Yamada, K., and Ahmed, S. 2018. Cytotoxic and anti-inflammatory compounds from Red Sea grass Thalassodendron ciliatum. Medicinal Chemistry Research, 27, 12381244.

Abdel-Wahhab, M.A., El-Nekeety, A.A., Hassan, N.S., El-Hefnawy, M.S., Kotb, M.M., El-Mekkawy, S.A., Khalil, N.A., and Hanna, A.G. 2012. Hepatoprotective effect of sarcophine isolated from soft coral (Sarcophyton glaucum) in rats. Global Veterinaria, 8, 244-253.

Bharate, S.B., Khan, S.I., Tekwani, B.L., Jacob, M., Khan, I.A., Singh, I.P. 2008. S-Euglobals: Biomimetic synthesis, antileishmanial, antimalarial, and antimicrobial activities. Bioorganic \& medicinal chemistry, 16, 1328-1336.

Di Pietro, M.E., Mannu, A., and Mele, A. 2020. NMR determination of free fatty acids in vegetable oils. Processes, 8, 410.

Iwagawa, T., Nakashima, R., Takayama, K., Okamura, H., Nakatani, M., Doe, M., and Shibata, K. 1999. New cembranes from the soft coral sarcophyton species. Journal of natural products, 62 , 1046-1049.

Chemistry, 15, 2593-2608.

Sun, B.N., Shen, H.D., Wu, H.X., Yao, L.X., Cheng, Z.Q., and Diao, Y. 2015. Determination of chemical 
Bioorganic \& medicinal chemistry, 13, 4458-4466.

Jia, R., Guo, Y.W., Mollo, E., Gavagnin, M., and Cimino, G. 2006. Sarcophytonolides E-H, cembranolides from the Hainan soft coral Sarcophyton latum. Journal of natural products, 69 , 819-822.

Lan, W.J., Li, H.J., Yan, S.J., Su, J.Y., and Zeng, L.M. 2007. New tetraterpenoid from the soft coral Sarcophyton tortuosum. Journal of Asian natural products research, 9, 267-71.

Pedpradab, P., Molex, W., and Suwanborirux, K. 2012. $3^{\text {rd }}$ International Conference on Chemistry and Chemical Engineering IPCBEE vol.38, IACSIT Press, Singapore.

Rahelivao, M.P., Lübken, T., Gruner, M., Kataeva, O., Ralambondrahety, R., Andriamanantoanina, H., Checinski, M.P., Bauer, I., and Knölker, H. 2017. Isolation and structure elucidation of natural products of three soft corals and a sponge from the coast of Madagascar. Organic \& Biomolecular constituents of the marine Pulmonate Slug, Paraoncidium reevesii. Tropical Journal of Pharmaceutical Research, 13, 2071.

Verseveldt, J. 1982. "A revision of the genus Sarcophyton Lesson (Octocorallia, Alcyonacea)" Brill.

Vichai, V., and Kirtikara, K. 2006. Sulforhodamine B colorimetric assay for cytoxicity screening. Nature protocols, 1, 1112-1116.

Wei Bie, Z.W. Deng, Z.W., Xu, M.J. and Lin, W.H. 2008. Structural elucidation of a new cembranoid diterpene from the Chinese soft coral Sarcophyton sp. Journal of Chinese Pharmaceutical Sciences, 17, 221-224.

Wei, W.C., Sung, P.J., Duh, C.Y., Chen, B.W., Sheu, J.H., and Yang, N.S. 2013. Anti-inflammatory activities of natural products isolated from soft corals of Taiwan between 2008 and 2012. Marine Drugs, 11, 4083-126. 